Gut, 1967, 8, 544

\title{
Morphological observations on gastric ulcers treated with carbenoxolone sodium ${ }^{1}$
}

\author{
T. E. W. GOODIER, L. HOR WICH, AND R. W. GALLOWAY \\ From St. Helier Hospital, Carshalton, Surrey, and Whiston Hospital, Prescot, Lancs.
}

EDITORIAL COMMENT These studies demonstrated increased mucus secretion and supported the hypothesis that this is a mechanism which plays a part in mucosal protection and repair.

Carbenoxolone, being a pentacyclic triterpene, is a drug which has a steroid-like structure, and despite antiinflammatory and mineralocorticoid-like effects resembling those of steroid has shown considerable success in producing healing of gastric ulceration (Doll, Hill, Hutton, and Underwood, 1962; Horwich and Galloway, 1965). The mode of action of carbenoxolone is not yet understood. The drug is rapidly absorbed from the stomach and it is believed to effect healing by a direct action on the gastric mucosa.

A possible explanation of the ability of carbenoxolone to heal gastric ulceration may lie in the observations at laparotomy that the carbenoxolonetreated gastric mucosa is covered by a remarkable amount of viscid, adherent mucus, whereas the gastric mucosa in patients treated with corticosteroids shows a decrease in the amount of visible mucus.

\section{MATERIAL}

This consists of specimens from four patients selected for partial gastrectomy for chronic gastric ulceration. The surgeon had agreed to treat these patients with carbenoxolone immediately before operation. All the patients had radiologically proven gastric ulceration which had failed to heal satisfactorily on dietary measures, rest, and alkalis.

\section{METHOD}

FIXATION OF SPECIMENS Immediately after partial gastrectomy, the stomach segment was wrapped in thin polythene and put in a vacuum flask containing solid carbon dioxide for transport to the laboratory, where it was fixed in a warm formaldehyde-C.P.C. solution (Conklin, 1963) for the better preservation of acid mucopolysaccharides.

${ }^{1}$ Carbenoxolone sodium is available under the brand name of Biogastrone and is abbreviated to carbenoxolone throughout this paper.
STAINING Paraffin sections of the ulcer and surrounding area were stained by haematoxylin and Biebrich scarlet, Alcian blue- P.A.S., P.T.A.H., aldehyde fuchsin, and M.S. B. All the partial gastrectomy specimens included the ulcer crater.

\section{CASE 1}

A woman, aged 31 years, had a two-year history of epigastric pain radiating to the back and intermittent vomiting. She was admitted with haematemesis and melaena following ingestion of four Phensic tablets. Her appetite was poor and she had lost $7 \mathrm{lb}$. in weight in the past seven months. The first haematemesis was one year previously. Blood group $\mathrm{A} R \mathrm{Rh}$ positive. Barium meal examination demonstrated an ulcer crater on the posterior wall of the pyloric antrum, $6 \mathrm{~mm}$. in diameter. Carbenoxolone, 50 mg. t.d.s., was given for 12 days immediately before the day on which the partial gastrectomy was performed.

PATHOLOGICAL EXAMINATION The specimen consisted of part of the pyloric antrum, containing a chronic ulcer $4 \mathrm{~mm}$. wide and $4 \mathrm{~mm}$. deep. Viscid mucus covered the surface of the gastric mucosa and partly filled the ulcer crater.

Histology The floor of the ulcer was covered by a $0.3 \mathrm{~mm}$. layer of Alcian- and P.A.S.-positive mucoid material mixed with fragments of surface epithelium, numerous mononuclear and pus cells, and a little fibrin. Below this layer there was a $0.4 \mathrm{~mm}$. zone of firmer, P.A.S.-positive fibrin, containing a few mononuclear and pus cells. Beneath this there was a layer of organizing, fine collagenous tissue, 4-5 mm. in thickness, with an Alcian- and aldehyde-fuchsin-positive matrix, containing mixed granulation tissue cells with many young capillaries and fibroblasts. The collagen progressively increased in density towards the muscle fibres. The surrounding mucosa showed considerable chronic inflammatory cellular exudate, including a few P.A.S.positive cells. The pyloric glandular cells, which formed the middle $2 \mathrm{~mm}$. of the lateral wall of the ulcer, were filled with P.A.S.-positive material, some of which was also free on the surface. The surface epithelial cells 
contained both Alcian-positive and P.A.S.-positive material. Proximally to the area of ulceration, the antral wall was heavily infiltrated by chronic inflammatory cells, including lymphoid nodules. There was more oedema than in the ulcer wall, especially in the submucosa, which showed some fibrin in the exudate, and increased collagen. There was increased P.A.S.-positive material in the deep glandular cells and neck cells, this material also escaping to the surface. There was a small amount of Alcianpositive material in the surface cells. Mast cells were evident in the mucosa, submucosa, and muscle. The fundal edge of the specimen showed similar chronic inflammatory changes in the slightly thickened mucosa. The neck cells and some deeper glandular cells contained P.A.S.-positive material. Parietal cells were prominent and zymogenic cells plentiful with normal granulation. The deeper mucosa showed a little interstitial fibrosis and the submucosa was oedematous. Mast cells were less numerous than near the ulcer.

\section{CASE 2}

A man, aged 38 years, had a history of epigastric pain relieved by alkalis for four years. He was admitted with haematemesis and melaena following ingestion of six Alka Seltzer tablets. A total of 7.5 litres of blood (group $\mathrm{O} \mathrm{Rh}$ positive) was transfused. Barium meal examination demonstrated a large ulcer crater in the pyloric antrum $2 \mathrm{~cm}$. in diameter. A pyloric stenosis was present with a large gastric residue 24 hours after the examination. Carbenoxolone, $100 \mathrm{mg}$. t.d.s., was given for four days before partial gastrectomy.

PATHOLOGICAL EXAMINATION The specimen consisted of the amputated pyloric antral part of the stomach, showing a chronic ulcer $18 \mathrm{~mm}$. in diameter and $12 \mathrm{~mm}$. deep. The central part of the ulcer floor was absent.

Histology The peripheral area of the ulcer floor consisted of a 1-2 mm. layer of fibrin mixed with necrotic mononuclear and pus cells. Beneath this was a 4-7 $\mathrm{mm}$. layer of oedematous fibrous tissue infiltrated by chronic inflammatory cells and containing endarteritic vessels. Its matrix stained weakly with Alcian blue and moderately with aldehyde fuchsin. Its vasculature and fibrogenesis appeared somewhat less active than in case 1 . The mucosa for the $15 \mathrm{~mm}$. adjacent to the ulcer showed a hyperplastic pattern, and mucous gland cells, both superficial and deep, stained intensely with P.A.S. More peripherally, increased P.A.S. reaction was not very obvious, the changes being those of chronic gastritis, but an occasional localized group of strongly P.A.S.positive secretory cells appeared on the surface amid some intestinalized epithelium.

\section{CASE 3}

A woman, aged 69 years, had a history of epigastric pain, relieved by dieting and alkalis, for 18 years. It was characterized by remissions and exacerbations, occasional vomiting and retching. Barium meal examination demonstrated an ulcer crater on the lesser curve of the stomach with associated spastic hourglass contracture. Gastroscopy confirmed these findings.

Carbenoxolone, $50 \mathrm{mg}$. t.d.s., was administered for six weeks before the operation with decrease in size of the ulcer crater as demonstrated by barium meal examination, from $8 \mathrm{~mm}$. to $5 \mathrm{~mm}$. in diameter.

PATHOLOGICAL EXAMINATION The ulcer crater was reported to have been filled with tenacious mucus, which was removed by the surgeon. The specimen consisted of a portion of the lesser curve of the stomach, showing a chronic ulcer, $6 \times 4 \mathrm{~mm}$. in diameter and $3 \mathrm{~mm}$. deep. The mucosa surrounding the ulcer appeared relatively firm and vascular to a diameter of $12 \mathrm{~mm}$.

Histology The ulcer penetrated the stomach wall to a depth slightly more than that of the surrounding mucosa. The floor of the ulcer consisted of a layer of organizing chronic inflammatory fibro-granulation tissue, the upper $0.3 \mathrm{~mm}$. being composed mainly of mononuclear and pus cells, the middle layer, of about $0.7 \mathrm{~mm}$., comprised dilated, young capillaries, growing towards the ulcer cavity and mixed with chronic inflammatory cells. The third layer consisted of organizing fibrous tissue, which extended to the subserosa, for a total of $5 \mathrm{~mm}$., almost completely replacing the muscle coat. There was also fibrosis of the submucosa for $5 \mathrm{~mm}$. surrounding the ulcer. Fibrin was scanty in the superficial part of the ulcer floor, and was mixed with P.A.S.-positive mucoid material at the edges. The mucosal glands in the $2 \mathrm{~mm}$. adjacent to the ulcer were irregular, suggestive of immaturity, without zymogenic or parietal cells, but showing production of varying amounts of P.A.S.positive material. Surface and neck cells contained similar P.A.S.-positive material. Alcian blue staining of epithelial cells was inconspicuous, although a normal amount appeared in the granulation tissue. More peripherally on either side the zymogenic and parietal cells of the gastric glands showed no obvious abnormality, but some neck cells appeared P.A.S.- positive. The mucosa surrounding the ulcer also contained a moderately heavy chronic inflammatory cellular exudate, including some lymphoid hyperplasia, and showed telangiectasia and oedema.

\section{CASE 4}

A woman, aged 52 years, had a vague history, but certainly several years of indigestion. A gastric ulcer had been demonstrated radiologically three years previously. She was admitted with an aspirin-induced melaena. Blood group $\mathbf{O} \mathbf{R}$ positive.

A barium meal examination demonstrated a large ulcer crater on the lesser curve of the stomach. Carbenoxolone was adminstered, $50 \mathrm{mg}$. t.d.s., for four weeks before partial gastrectomy. A repeat barium meal two days before the operation showed marked decrease in the size of the ulcer from $2.0 \mathrm{~cm}$. to $5 \mathrm{~mm}$. in diameter.

PATHOLOGICAL EXAMINATION The specimen consisted of a portion of stomach showing a chronic ulcer on the lesser curve, which measured $3 \mathrm{~mm}$. in width and $4 \mathrm{~mm}$. in depth. There was puckering of the mucosa around the 
ulcer, compatible with healing, and the appearance of the mucosa generally suggested chronic gastritis.

Histology The ulcer crater contained P.A.S.-positive mucoid material mixed with a little fibrin. The floor consisted of a mass of inflammatory cells, below and on either side of which there was vascular granulation tissue, then young fibrous tissue having an Alcianpositive matrix, and finally an extensive area of more collagenous scar. This scar measured on the slide $16 \mathrm{~mm}$. in width, which, allowing for shrinkage during dehydration, suggested a scar in the original specimen of some $25 \mathrm{~mm}$., giving an estimated healing of the ulcer by $85 \%$. The side walls of the ulcer crater consisted of regenerating mucosa of normal thickness, in which epithelial cells were distended with P.A.S.-positive material and the irregular pits were filled with similar material, which also covered the surface of the mucosa. The zone of neck cells at the ulcer edge appeared deeper than normal. The surrounding mucosa showed considerable chronic inflammatory cellular exudate, with reactive lymphoid nodules, and the glands showed a high proportion of P.A.S.-positive epithelial cells in the neck regions.

\section{DISCUSSION}

Although in three out of the four patients, bleeding was precipitated by ingestion of aspirin, none of the patients was taking aspirin routinely.

Histological sections inevitably provide very small samples of tissue, and data from them have to be interpreted with reserve, since the sample may not be representative of the whole organ. In addition there is shrinkage of the tissue to about $70 \%$ during the process of dehydration for paraffin sections. In gastric histology there is certainly variation, both in different areas of the same stomach and in the same area of different stomachs, and the solubility of mucus in watery fixatives is a problem. However, with the support of macroscopic findings, even from an extremely small series such as the present, it is possible to draw the following tentative conclusions:-

1 Gastric ulcers treated by oral carbenoxolone undergo healing of a basically normal pattern, by fibrosis of the ulcer floor, and epithelial ingrowth from the edges.

2 Fibroblastic activity, collagen production, capillary growth, and inflammatory cellular exudate appear unimpaired by the drug.

3 Acid mucopolysaccharide content of young fibrous tissue is not obviously reduced.

4 The fibrin barrier in the ulcer floor is not increased in density.

5 The ulcer floor is covered by a layer of P.A.S.positive mucinous material.

6 Regenerating epithelium at the ulcer edge and sides soon contains P.A.S.-positive material.

7 The mucous cells of the pits and the pit cavities in the region of the ulcer contain abundant P.A.S.positive material.

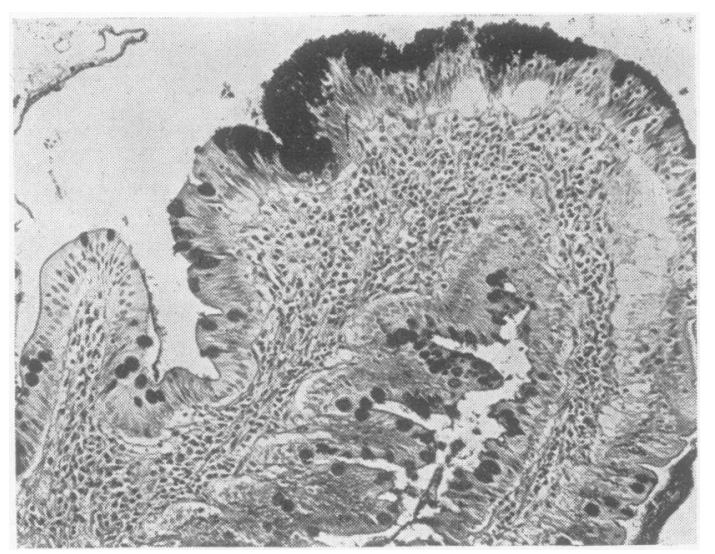

FIG. 1. (Alcian-P.A.S.-haematoxylin $\times 100$ ). Case 2 (antral ulcer). Antral mucosa showing gastritis with intestinalization. An area of increased P.A.S.-positive secretion by the surface epithelium stands out from the adjacent and deeper goblet-celled pattern.

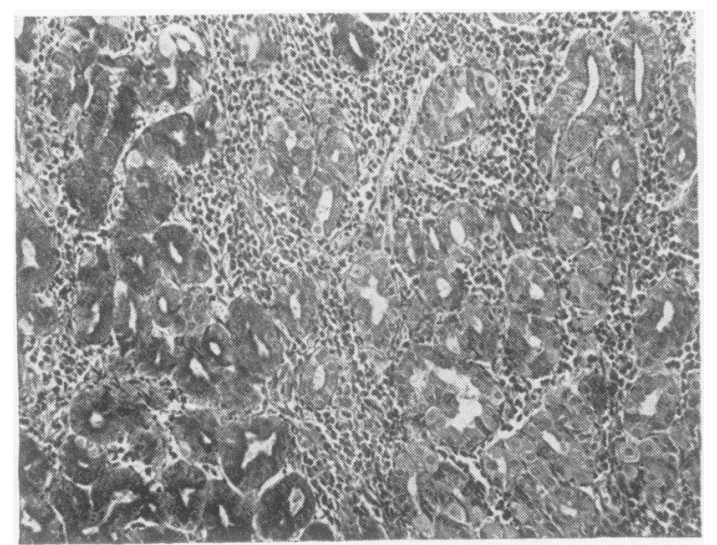

FIG. 2. (Alcian-P.A.S.-haematoxylin $\times 120$ ). Case 3 (lesser curve ulcer). Mucosa at side of ulcer, just below 'neck' level. Glands nearer ulcer (left) are composed largely of darker P.A.S.-positive cells. Remaining glands (right) show a more normal distribution of cells.

8 Proliferative (intestinalized) mucosa near an ulcer show P.A.S.-positive secretion by the surface cells (Fig. 1).

9 Neck cells away from the ulcer show an increased tendency to be P.A.S.-positive (Fig. 2).

It may be assumed for practical purposes that the main proportion of P.A.S.-positive material in the gastric mucosa consists of epithelial neutral mucopolysaccharide, suggesting that with carbenoxolone those cells capable of secreting epithelial mucin produce a greater amount of mucus, including early conversion of regenerated epithelial cells to mucin production. Carbenoxolone itself shows no staining reaction to P.A.S. 
There is a fair amount of experimental evidence in animals to support the historical concept that the gastric mucosa can cover itself with a coat of mucus, which acts as protection against ulceration. Working with rats and dogs, Robert and Nezamis (1963), Robert, Bayer, and Nezamis (1963), Robert and Nezamis (1964), Menguy and Masters (1963), and Menguy and Masters (1965a) have shown that drugs which reduce the amount of mucus in the gastric juice and mucosa tend to increase the liability to gastric ulceration, and conversely the same authors, Robert and Nezamis (1964) and Menguy and Masters (1965b), have also provided evidence that drugs with which there is associated an increase in gastric juice mucus and mucosal mucus reduce gastric ulceration. The work of Doberneck and Engle (1966) indicates that the human gastric mucosa in cases of gastric ulcer (and also of chronic duodenal ulcer with pyloric obstruction) does, in fact, approximately double its number of mucussecreting cells in the grossly normal area away from any lesion. Carbenoxolone tends to facilitate the normal healing process in gastric ulcers as far as can be seen by routine histological methods; in addition, the macroscopic observation of increased surface mucus, coupled with the microscopic finding of an abundance of P.A.S.-positive mucinous material in the mucosal pits and cells in the vicinity of carbenoxolone-treated gastric ulcers, support the hypothesis that one of the factors is an increased secretion of mucus by the gastric epithelium and particularly by regenerating epithelial cells in the vicinity of the healing ulcer. It seems reasonable to suppose that increased production of mucin by the gastric epithelium is a physiological response playing a part in mucosal protection and repair.

\section{SUMMARY}

Four patients with chronic gastric ulcer are des- cribed, who were given oral carbenoxolone before undergoing partial gastrectomy. The ulcers appeared to have healed to some extent, and it is suggested that an increased production of mucin by the gastric mucosa may have assisted in the healing process.

We gratefully acknowledge the assistance of Mr. J. A. Martinez who supplied the operative specimens, of Dr. S. Gottfried, Biorex Laboratories, Ltd., who organized their transport and provided secretarial help, and of Mr. Finch, Department of Anatomy, Guy's Hospital, in the preparation of the photomicrographs.

REFERENCES

Conklin, J. L. (1963). Staining reactions of mucopolysaccharides after formalin-containing fixatives. Stain Technology, 38, 56-59.

Doberneck, R. C., and Engle, J. C. (1966). Quantitative evaluation of the gastric mucosa of normal subjects and subjects with various gastric disorders. Surgery, 59, 189-194.

Doll, R., Hill, I. D., Hutton, C., and Underwood, D. J., II (1962). Clinical trial of a triterpenoid liquorice compound in gastric and duodenal ulcer. Lancet, 2, 793-796.

Horwich, L., and Galloway, R. (1965). Treatment of gastric ulceration with carbenoxolone sodium: clinical and radiological evaluation. Brit. med. J., 2, 1274-1277.

Menguy, R., and Masters, Y. F. (1963). Effect of cortisone on mucoprotein secretion by gastric antrum of dogs; pathogenesis of steroid ulcer. Surgery, 54, 19-28.

- - (1965a). Effects of aspirin on gastric mucous secretion. Surg. Gynec. Obstet., 120, 92-98.

- - - (1965b). Influence of parathyroid extract on gastric mucosal content of mucus. Gastroenterology, 48, 342-349.

Robert, A., and Nezamis, J. E. (1963). Effect of prednisolone on gastric mucus content and on ulcer formation. Proc. Soc. exp. Biol. (N.Y.), 114, 545-550.

- Bayer, R. B., and Nezamis, J. E. (1963). Gastric mucus content during development of ulcers in fasting rats. Gastroenterology, 45, 740-751.

- and Nezamis, J. E. (1964). Effect of anti-acetylcholine drug, methscopolamine bromide, on ulcer formation and gastric mucus. J. Pharm. Pharmacol., 16, 690-695. 